\title{
Dinámica del carbono lábil del suelo en sistemas forestales de Eucalyptus grandis Hill ex Maiden en la Mesopotamia Argentina
}

\author{
Dynamic of soil labile carbon in forest systems \\ of Eucalyptus grandis Hill ex Maiden in the Argentinean Mesopotamia
}

\author{
Romina I Romaniuk a*, Matias Venece ${ }^{\mathrm{b}}$, Vanina R N Cosentino ${ }^{\text {a,c }}$, Carina R Alvarez ${ }^{\text {b, }}$ \\ Esteban A Ciarlo d, Helena Rimski Korsakov ${ }^{b}$, Haydée S Steinbach ${ }^{b}$, Ana M Lupi ${ }^{a}$ \\ *Autor de correspondencia: a Instituto Nacional de Tecnología Agropecuaria (INTA), Instituto de Suelos, \\ Nicolás Repetto y de Los Reseros s/n, CP 1686, Hurlingham, Buenos Aires, Argentina, tel.:+54 (11) 46211448, \\ romaniuk.romina@inta.gob.ar \\ b Universidad de Buenos Aires, Facultad de Agronomía, Cátedra de Fertilidad y Fertilizantes, CABA, Argentina. \\ ${ }^{c}$ Consejo Nacional de Investigaciones Científicas y Técnicas, CABA, Argentina. \\ d Universidad de Buenos Aires, Facultad de Agronomía, Cátedra de Edafología, CABA, Argentina.
}

\begin{abstract}
SUMMARY
The amount and quality of carbon from forest litter present in the soil will depend on several factors, including species composition, management practices, plantation age, climatic conditions and soil texture, among several other factors. The aims of this research were to: i) quantify the labile organic carbon content in soils with Eucalyptus grandis plantations of the Mesopotamian region in Argentina and evaluate its relationship with the amount and type of litter; and ii) identify management, edaphic and environmental factors that may influence its variation. The study was performed on young and middle age plantations having soils with sandy and clayey texture located in the Gualeguaychú district, Entre Ríos, Argentina. To quantify the labile carbon in the soil, the soluble organic carbon was extracted with a $0.5 \mathrm{M}$ potassium sulfate solution. Soluble organic carbon concentration did not differ according to the age of the stands, with average values of $45.6 \mathrm{mg} \mathrm{kg}^{-1}$ in clay soils, and of $32.8 \mathrm{mg} \mathrm{kg}^{-1}$ in sandy soils, showing a positive association with clay content. The highest soluble organic carbon values were recorded during the summer months, and were positively related to the increases in the water-saturated pore space and the soil biological activity. We also verified a linear and positive relationship between the amount of initial fine litter and the increase of soluble organic carbon in the soil $\left(\mathrm{R}^{2}=0.41\right)$. The reported results provide explicit ranges of variability for total organic carbon, soluble organic carbon and carbon fluxes at local level; thereby contributing to further characterize carbon modeling in forest systems.
\end{abstract}

Key words: Litter, sandy and clay soils.

\section{RESUMEN}

La cantidad y calidad de carbono del mantillo forestal que ingresa al suelo depende de varios factores, entre ellos la especie implantada, el manejo, edad de la plantación, las condiciones climáticas y la textura del suelo. Los objetivos de este trabajo fueron i) cuantificar el contenido de carbono orgánico lábil en suelos con plantaciones Eucalyptus grandis (Eucalyptus grandis) de la región mesopotámica y evaluar su relación con la cantidad y tipo del mantillo; e ii) identificar aquellos factores de manejo, edáficos y ambientales que influyen en su variación. Se trabajó sobre plantaciones jóvenes y de mediana edad en suelos de textura arenosa y arcillosa localizados en el Departamento de Gualeguaychú, Entre Ríos, Argentina. Con el fin de cuantificar el carbono lábil del suelo, se procedió a extraer el carbono orgánico soluble con una solución de sulfato de potasio $0,5 \mathrm{M}$. Los valores de carbono orgánico soluble no difirieron según la edad de los rodales, con valores promedio de $45,6 \mathrm{mg} \mathrm{kg}^{-1}$ en los suelos arcillosos, y de $32,8 \mathrm{mg} \mathrm{kg}^{-1}$ en los arenosos, mostrando una asociación positiva con el contendido de arcilla. Los mayores valores de carbono soluble se registraron durante los meses estivales, relacionándose positivamente con los incrementos en el espacio poroso saturado con agua y con la actividad biológica del suelo. Pudo verificarse una relación lineal y positiva entre la cantidad de mantillo fino inicial y el incremento de carbono orgánico soluble en el suelo $\left(\mathrm{R}^{2}=0,41\right)$. Los resultados reportados cuantifican las existencias de carbono orgánico total, carbono orgánico soluble y flujos de carbono a nivel local, contribuyendo con información necesaria para el modelado del carbono en sistemas forestales.

Palabras clave: mantillo, suelos arenosos, suelos arcillosos. 


\section{INTRODUCCIÓN}

Los sistemas forestales desempeñan un papel central en el ciclo del carbono, ya que lo capturan de la atmósfera a medida que crecen, almacenándolo en sus tejidos, acumulando gran cantidad de biomasa. A su vez, estos sistemas poseen un gran potencial para almacenar carbono orgánico en el suelo, y, de esta manera, pueden contribuir a mitigar el calentamiento global (Montero 2005). En Argentina, las plantaciones forestales cubren aproximadamente 1.300 .000 ha, de las cuales el $24 \%$ son de Eucalyptus spp., con 112.000 ha ubicadas en la Provincia de Entre Ríos (MAGyP 2020).

Las variaciones en los contenidos de carbono orgánico total suelen verse en el largo plazo. Según el panel intergubernamental de cambio climático (IPCC 2006) el lapso de tiempo requerido para observar cambios en el almacenaje de carbono orgánico total en los suelos bajo un determinado manejo del cultivo no debería ser menor a 20 años, ya que los cambios son graduales y solo cuantificables luego de un largo período. Por el contrario, debido a su naturaleza dinámica, las fracciones lábiles de materia orgánica pueden responder rápidamente a los cambios en las entradas de carbono al sistema (Haynes 2000). El carbono lábil del suelo está principalmente constituido por compuestos orgánicos simples, aminoácidos, y carbohidratos entre otros compuestos, lo que facilita el acceso y transformación por parte de los microorganismos del suelo (Zou et al. 2005). A pesar que el carbono lábil representa solo una pequeña fracción del carbono orgánico total de los suelos, puede influir notablemente en la actividad biológica (Chen y Xu 2005). Dentro de las formas de carbono lábil, el carbono orgánico soluble extraído con sulfato de potasio $0,5 \mathrm{M}$ puede ser utilizado como una medida de la fracción ligera de materia orgánica total del suelo que es fácilmente degradable por los microorganismos, y que, por tanto, está estrechamente relacionada con la actividad y el crecimiento microbiano (Hofman y Dusek 2003). El carbono orgánico soluble proviene principalmente de productos solubles mono u oligoméricos de descomposición microbiana y de aportes vegetales como exudados de raíces y lixiviados de hojas que contribuyen activamente a la dinámica de la materia orgánica en los suelos minerales (Abramoff et al. 2018). Por otro lado, puede ser asimilado por los microorganismos que actúan como consumidores y productores de esta forma de carbono. A su vez, el carbono orgánico soluble es el principal dador de electrones durante la denitrificación, proceso responsable de la generación y emisión a la atmósfera de formas gaseosas de nitrógeno, entre ellas el óxido nitroso, potente gas de efecto invernadero. En este sentido ha sido registrado que los residuos que se incorporan al suelo proveen carbono orgánico soluble que estimula los procesos de denitrificación y emisión de óxido nitroso (Millar y Baggs 2004).

Una de las características distintivas de la mayor parte de los ecosistemas forestales es el desarrollo de una cubier- ta orgánica que resulta del retorno periódico, a través de la caída de las hojas, ramas, corteza, frutos y a veces árboles completos. Esta acumulación de restos orgánicos, llamada hojarasca, necromasa o mantillo, retiene una gran proporción de carbono, fijado por fotosíntesis, y de nutrientes extraídos del suelo por los árboles (Sayer 2006). Las reservas de carbono orgánico total de los suelos forestales dependen de la entrada de carbono desde el mantillo, por lo que su descomposición juega un rol central en los ciclos biogeoquímicos de los nutrientes y en la formación de nueva materia orgánica en los suelos (Hättenschwiler et al. 2005). La actividad de los organismos descomponedores está regulada por las condiciones edafoclimáticas (van Diepen et al. 2015). Según la interacción entre estas condiciones durante la descomposición del mantillo parte del carbono es convertido a $\mathrm{CO}_{2}$, mientras que otra parte es incorporada en distintas fracciones de carbono orgánico total, pudiendo pasar a conformar parte de la biomasa microbiana para luego convertirse en carbono estable o en carbono soluble, estando este último expuesto a lixiviarse (Leifeld y KögelKnabner 2005). Sin embargo, estudios recientes indican que la mayoría del carbono soluble proveniente del mantillo es retenida por el suelo (Wang et al. 2019 a).

El mantillo que cubre los suelos o piso forestal varía en cantidad y composición de acuerdo con numerosos factores, tales como la edad de los rodales, el manejo del sistema silvícola, el clima, y el tipo de suelo. Su tasa de descomposición dependerá de la calidad y estructura de la fuente carbonada, y de las condiciones del medio, como la humedad y la temperatura (Silveira et al. 2011). Rodales maduros, que ya han llegado a su máximo crecimiento comercial o edad de rotación, probablemente posean mayor cantidad de mantillo, estando a su vez compuesto por mayor proporción de material lignificado que el que podría encontrarse en rodales jóvenes. Sin embargo, la cantidad y calidad del mantillo en un rodal joven dependerá del uso previo del lote (forestal, pastura, campo natural) o de las prácticas utilizadas en preparación del terreno, y manejo de residuos de cosecha en superficie, entre otros. A su vez, la textura de los suelos puede influir en el ciclado de este mantillo y en la formación y conservación de la materia orgánica del suelo y sus fracciones. Los suelos arcillosos, en comparación con aquellos de textura arenosa, tienen una mayor cantidad de biomasa microbiana que puede acelerar el proceso de transformación de carbono desde el mantillo (Goya et al. 2008). La materia orgánica derivada del mantillo puede asociarse con las arcillas y estabilizarse por protección física (McLauchlan 2006). Por su parte, en los suelos arenosos, con menor actividad microbiana, la descomposición y transformación del mantillo suele ser más lenta, estando a su vez el carbono soluble más expuesto a perderse por lixiviación (Kalbitz et al. 2000).

Si bien algunos autores han estudiado la dinámica de liberación de nutrientes y carbono desde el mantillo de $E u$ calyptus spp. en diversos suelos y ambientes en Argentina (Goya et al. 2008) y otros países (Wang et al. 2019 b), 
De Lucena et al. (2019) sostienen que aún son pocas las investigaciones acerca del efecto de las prácticas forestales sobre la acumulación y depósito de carbono en el suelo. Sumado a ello, existe una tendencia a que gran parte de los investigaciones que estudian la dinámica del carbono orgánico soluble en suelos forestales sean realizados en condiciones controladas de laboratorio, siendo escasos aún los estudios in situ, motivo por el cual Kalbitz et al. (2000) puntualizan en la necesidad de realizar investigaciones a campo. Generar esta información para sistemas forestales representativos de la región mesopotámica permitiría contar con valores base de las existencias de carbono del suelo, necesarios para el modelado del carbono en sistemas forestales. En este contexto, el presente trabajo busca responder las siguiente pregunta: ¿Cómo influyen la edad de la plantación, las prácticas silvícolas y las condiciones edafoclimáticas en los componentes del mantillo y en el carbono lábil del suelo? En base a ello, los objetivos de esta investigación fueron i) cuantificar el contenido de carbono orgánico soluble en suelos con plantaciones de $E u$ calyptus grandis Hill. ex Maiden de la región mesopotámica y evaluar su relación con la cantidad y tipo del mantillo; e ii) identificar aquellos factores de manejo, edáficos y ambientales que influyen en su variación.

\section{MÉTODOS}

Sitio de estudio. El sitio de estudio se encuentra en el Departamento de Gualeguyachú, provincia de Entre Ríos, Argentina $\left(33^{\circ} 1^{\prime} 17^{\prime \prime} \mathrm{S} ; 58^{\circ} 13^{\prime} 37^{\prime \prime} \mathrm{W}\right)$. El clima predo- minante es del tipo templado húmedo de llanura, presentando una temperatura media anual de $17,9^{\circ} \mathrm{C}$, siendo la temperatura media máxima de $23,6^{\circ} \mathrm{C}$ y la media mínima de $12,2^{\circ} \mathrm{C}$. La precipitación media anual asciende a 1.100 $\mathrm{mm}$ (Forte Lay et al. 2008).

Se trabajó en plantaciones de E. grandis Hill ex Maiden en estadios pre y post cierre de copa, tratamientos. Para ello se utilizaron rodales jóvenes (entre uno y tres años) y de mediana edad (entre siete y nueve años). A su vez, las plantaciones se ubicaron en suelos de diferentes texturas, arenosa (ARE) correspondiente a un suelo Udifluvent Oxico, y arcillosa (ARC), clasificado como Peludert Argílico (Soil Survey Staff, 2014). En cada uno de estos suelos se seleccionaron tres rodales con plantaciones jóvenes, y tres lotes con plantaciones de mediana edad, con densidades de plantación de entre 700 y 850 plantas por ha. La caracterización edáfica y forestal de las situaciones seleccionadas se presenta en el cuadro 1.

En la profundidad de 0 a $5 \mathrm{~cm}$ se determinaron la textura del suelo (Ashworth et al. 2001), el pH por potenciometría (relación suelo/agua: 1:2,5), la densidad aparente (DA) por el método del cilindro (volumen de $100 \mathrm{~cm}^{3}$ ) (cuadro 1) y el carbono orgánico total (COT) por Walkley y Black (Nelson y Sommers 1996) (Figura 3).

Para el área de estudio, el crecimiento histórico promedio, en términos de producción de biomasa, es de 25 y $35 \mathrm{~m}^{3} \mathrm{ha}^{-1}$ año $^{-1}$ para las plantaciones instaladas en suelos arcillosos y arenosos, respectivamente (comunicación personal). Los datos meteorológicos durante el período de estudio se presentan en la figura 1.

Cuadro 1. Valores medios y error estándar de las variables analizadas para la caracterización edáfica $(0-5 \mathrm{~cm})$ y forestal de las situaciones en estudio. ARC: suelo arcilloso, ARE: suelo arenoso, J: rodal joven, M: rodal de mediana edad. COT: carbono orgánico total, DA: densidad aparente del suelo, DP: densidad de plantas, DAP diámetro del tronco a la altura del pecho.

Mean values and standard error of the soil variables analyzed $(0-5 \mathrm{~cm})$ and forest characterization of the study situations. ARC: clayey soil, ARE: sandy soil, J: young stands, M: middle-age stands. COT: total soil organic carbon, DA: soil bulk density, DP: plant density, DAP: diameter at breast height.

\begin{tabular}{|c|c|c|c|c|c|c|c|c|c|c|c|c|}
\hline Variable & \multicolumn{3}{|c|}{ ARC J } & \multicolumn{3}{|c|}{ ARC M } & \multicolumn{3}{|c|}{ ARE J } & \multicolumn{3}{|c|}{ ARE M } \\
\hline \multicolumn{13}{|c|}{ Caracterización de suelos } \\
\hline Arena $(\%)$ & 41,83 & \pm & 8,85 & 31,67 & \pm & 1,74 & 74,50 & \pm & 3,04 & 91,17 & \pm & 0,83 \\
\hline Limo (\%) & 31,33 & \pm & 5,64 & 35,33 & \pm & 2,42 & 15,00 & \pm & 2,08 & 4,67 & \pm & 0,83 \\
\hline Arcilla (\%) & 26,83 & \pm & 4,04 & 33,00 & \pm & 2,93 & 10,50 & \pm & 1,04 & 4,17 & \pm & 0,83 \\
\hline $\mathrm{pH}(1: 2,5)$ & 6,27 & \pm & 0,37 & 6,10 & \pm & 0,32 & 5,63 & \pm & 0,09 & 5,57 & \pm & 0,07 \\
\hline DA $\left(\mathrm{g} \mathrm{cm}^{-3}\right)$ & 1,23 & \pm & 0,03 & 1,20 & \pm & 0,07 & 1,26 & \pm & 0,03 & 1,30 & \pm & 0,04 \\
\hline \multicolumn{13}{|c|}{ Caracterización forestal } \\
\hline Año de plantación & \multicolumn{3}{|c|}{2013} & \multicolumn{3}{|c|}{2007} & \multicolumn{3}{|c|}{2015} & \multicolumn{3}{|c|}{2009} \\
\hline Historia previa & \multicolumn{3}{|c|}{ Campo natural } & \multicolumn{3}{|c|}{ Campo natural } & \multicolumn{3}{|c|}{ Pino (1999-2015) } & \multicolumn{3}{|c|}{ Campo natural } \\
\hline DP (plantas ha-1) & 858 & \pm & 153 & 693 & \pm & 66,0 & 864 & \pm & 31,0 & 864 & \pm & 70,0 \\
\hline Altura media (m) & 9,97 & \pm & 2,94 & 21,80 & \pm & 0,95 & 5,60 & \pm & 1,06 & 22,57 & \pm & 0,50 \\
\hline DAP medio $(\mathrm{cm})$ & 11,37 & \pm & 1,67 & 18,20 & \pm & 0,75 & 5,40 & \pm & 1,05 & 19,0 & \pm & 0,55 \\
\hline
\end{tabular}




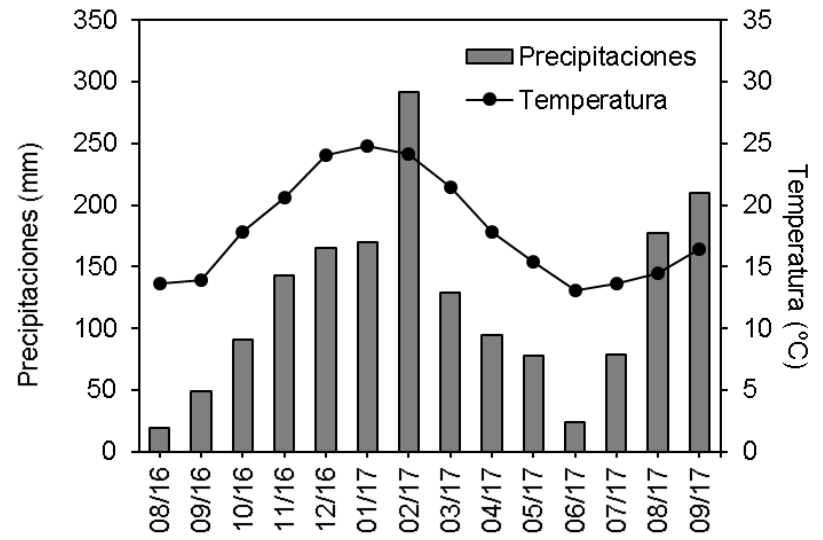

Figura 1. Precipitaciones mensuales acumuladas y temperaturas medias del aire durante el período de estudio.

Accumulated monthly rainfall and mean air temperatures during the studied period.

Muestreo y determinaciones. El mantillo acumulado en la superficie de los suelos se recolectó al inicio del estudio, en agosto de 2016. Para ello, en cada rodal se establecieron tres parcelas circulares, con un radio de $12 \mathrm{~m}$. En cada parcela se tomaron cuatro muestras de mantillo al azar, en una superficie de $0,25 \mathrm{~m}^{2}$. Una vez en laboratorio, el material se separó en dos categorías. A) Grueso: compuestos por restos lignificados de ramas, corteza y frutos. B) Fino: compuesto por hojas de la plantación y material herbáceo del sotobosque (vivo o muerto). Todas las muestras se secaron en estufa a $65^{\circ} \mathrm{C}$ hasta peso contante.

Se evaluó mensualmente el contenido de carbono soluble en los primeros $5 \mathrm{~cm}$ de suelo para los distintos tratamientos a lo largo de un año. El primer muestreo se realizó a finales del mes de agosto de 2016. Por problemas de acceso al establecimiento, no pudieron realizarse los muestreos de noviembre de 2016 y julio de 2017, por lo que, para completar los doce muestreos, el esquema original de un año se extendió a 14 meses desde la fecha de inicio.

Para la determinación del carbono soluble se procedió según la metodología propuesta por Vance et al. (1987). También se determinaron en cada muestreo, el contenido de humedad gravimétrica, densidad aparente del suelo y temperatura del aire y del suelo. Para la determinación la humedad gravimétrica $(\mathrm{Hg})$ las muestras de suelo fueron secadas en estufa a $110^{\circ} \mathrm{C}$ hasta constancia de peso. La densidad aparente se determinó por el método del cilindro citado previamente. Considerando la humedad gravimétrica y la densidad aparente se calculó el espacio poroso saturado con agua (EPSA) según la ecuación 1.

$$
\operatorname{EPSA}(\%)=\frac{(H g * D A * 100)}{\left[1-\left(\frac{D A}{2,65}\right)\right]}
$$

Donde: EPSA = espacio poroso saturado con agua (\%); $\mathrm{Hg}=$ humedad gravimétrica $\left(\mathrm{g} \mathrm{g}^{-1}\right)$; $\mathrm{DA}=$ densidad aparente $\left(\mathrm{g} \mathrm{cm}^{-3}\right)$.
En todas las fechas que se midió el carbono soluble se determinó la tasa de emisión de $\mathrm{CO}_{2}$ como indicador de la actividad biológica, reflejando fundamentalmente, la respiración de los microorganismos del suelo. La determinación del $\mathrm{CO}_{2}$ se realizó mediante la utilización de dos cámaras estáticas ubicadas aleatoriamente en cada parcela. Cada cámara consistió en una bandeja invertida de PVC de $16,66 \mathrm{dm}^{3}(0,43 \times 0,31 \times 0,125 \mathrm{~m})$. Las muestras de aire se extrajeron con jeringas y se inyectaron en viales de 10 $\mathrm{ml}$, asegurando la purga del aire contenido previamente en los mismos. Se tomaron muestras a los 0,15 y 30 minutos en cada cámara luego de su cierre, y a partir de ellas se calculó la tasa de emisión. Las muestras gaseosas fueron analizadas mediante cromatografía de gases (Agilent Network GC System, AECD, Santa Clara, USA).

Análisis estadístico. Los resultados se analizaron mediante análisis de varianza convencionales (ANOVA) y comparación de medias (prueba de LSD) con un nivel de significancia de $P=0,05$. Previo al ANOVA se corroboraron los supuestos de normalidad de los datos. Se realizaron correlaciones entre variables y se ajustaron modelos de regresión simple.

\section{RESULTADOS}

Caracterización del mantillo y del carbono orgánico total $y$ soluble del suelo. No se observaron diferencias significativas entre tratamientos en la cantidad de materia seca total del mantillo ( $P=0,21$; figura 2$)$ ni en la fracción gruesa del mismo $(P=0,37)$. Por otro lado, sí se observaron diferencias $(P<0,05)$ en el mantillo fino, presentando el tratamiento con rodales de mediana edad en suelo arenoso valores significativamente mayores respecto al tratamiento con rodales jóvenes en suelo arcilloso.

Independientemente de la edad del rodal, los suelos arcillosos presentaron mayores contenidos de carbono orgánico total (figura 3) a pesar de mostrar contenidos similares de materia seca total en el mantillo (figura 2). Por otro lado, dentro de los suelos arenosos, el tratamiento con rodales de mediana edad tuvo menores valores de carbono orgánico total que el tratamiento con rodales jóvenes.

Los valores de concentración de carbono orgánico soluble al inicio del ensayo variaron entre 33 y $66 \mathrm{mg} \mathrm{kg}^{-1}$, con un valor promedio de $40 \mathrm{mg} \mathrm{kg}^{-1}$ en los suelos arcillosos, y entre 18 y $30 \mathrm{mg} \mathrm{kg}^{-1}$, con un promedio de $24 \mathrm{mg}$ $\mathrm{kg}^{-1}$ en los suelos arenosos (figura 3). En general, el carbono soluble presentó un patrón similar al carbono orgánico total. Mientras que los valores de carbono orgánico total para ambos tratamientos instalados en los suelos arcillosos (ARC J y ARC M) fueron significativamente mayores que los observados en los suelos arenosos (ARE J y ARE M), las concentraciones de carbono orgánico soluble solo fueron significativamente mayores para el tratamiento con rodales jóvenes en suelo arcilloso (ARC J) en relación a las plantaciones jóvenes y maduras en suelos arenosos. Tanto el carbono orgánico total como el carbono soluble inicial 
se relacionaron positivamente con el contenido de arcilla $(\mathrm{R}=0,52 P<0,001$ y $\mathrm{R}=0,11 P<0,01$, respectivamente).

Dinámica del carbono soluble en el tiempo. En la figura 4 se puede observar, para todos los tratamientos, un aumento gradual del carbono soluble hasta llegar a un pico en los meses estivales, coincidiendo con los máximos valores registrados de precipitación y temperatura en la zona (figura 1),

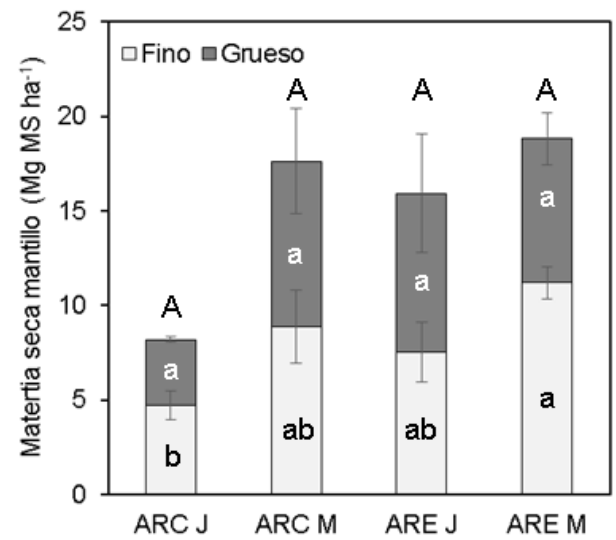

Figura 2. Materia seca de mantillo grueso y fino para cada tratamiento. ARC: suelo arcilloso, ARE: suelo arenoso, J: rodal joven, M: rodal de mediana edad. Letras diferentes indican diferencias significativas entre tratamientos $(P<0,05)$. Las letras mayúsculas indican las diferencias en el mantillo total y las letras minúsculas comparan las diferencias para las fracciones de mantillo grueso y fino.

Coarse and fine litter dry matter for each treatment. ARC: clayey soil, ARE: sandy soil, J: young stands, M: middle-age stands. Different letters indicate significant differences among treatments $(P<$ 0.05). Capital letters indicate the differences in total mulch and lower case letters compare the differences for the coarse and fine litter fractions. para luego mantener una tendencia estable a lo largo del tiempo. En general, puede observarse mayores valores de carbono soluble en los suelos arcillosos que en los arenosos, a excepción de los primeros meses debido a la alta variabilidad en los datos en el tratamiento con rodales jóvenes sobre suelo arcilloso. Para los suelos de ambas texturas, no se observaron diferencias según la edad de los rodales. Los valores de carbono orgánico soluble promedio considerando todo los tiempos de muestreo fueron de 45,6 $\mathrm{mg} \mathrm{kg}^{-1}$ para los suelos arcillosos, y de $32,8 \mathrm{mg}$

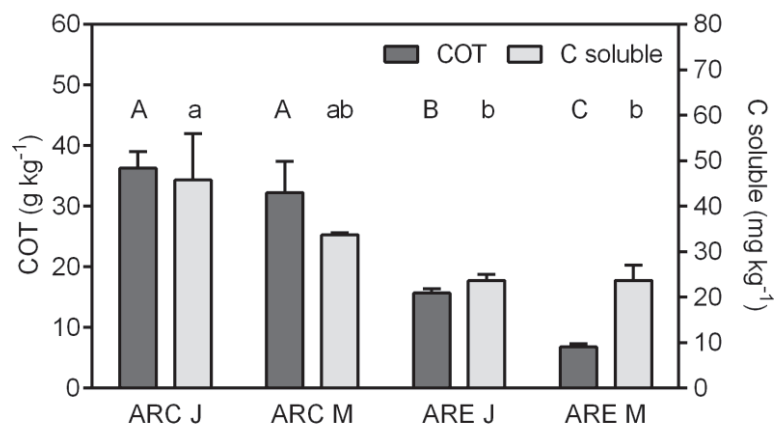

Figura 3. Valores medios y error estándar de carbono orgánico total (COT) y carbono orgánico soluble inicial (C soluble). Letras diferentes mayúsculas indican diferencias del COT entre tratamientos $(P<0,05)$ y letras diferentes minúsculas indican diferencias del $\mathrm{C}$ soluble entre tratamientos $(P<0,05)$. ARC: suelo arcilloso, ARE: suelo arenoso, J: rodal joven, M: rodal de mediana edad.

Mean values and standard error of soil total organic carbon (COT) and initial soluble carbon (C soluble). Different capital letters indicate differences in COT among treatments $(P<0.05)$ and different lower case letters indicate differences in soluble $\mathrm{C}$ among treatments $(P<0.05)$. ARC: clayey soil, ARE: sandy soil, J: young stand, M: middle-aged stand.

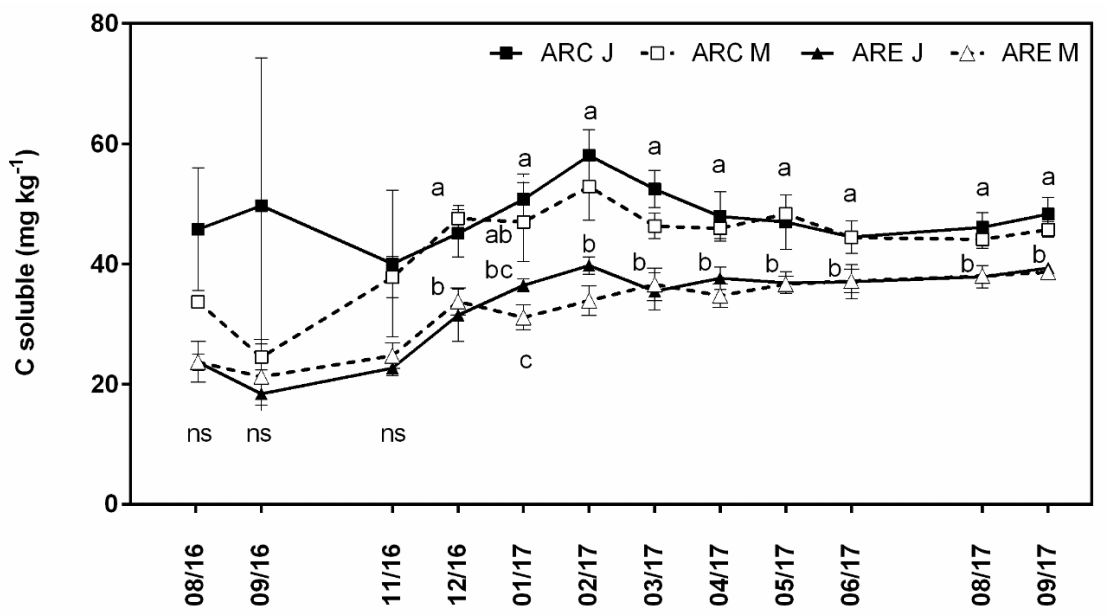

Figura 4. Evolución del carbono orgánico soluble (C soluble) en el tiempo. ARC: suelo arcilloso, ARE: suelo arenoso, J: rodal joven, M: rodal de mediana edad. Letras diferentes indican diferencias significativas entre tratamientos dentro de la misma fecha de muestreo $(P<0,05)$.

Evolution of soluble carbon (C soluble) over time. ARC: clayey soil, ARE: sandy soil, J: young stand, M: middle-aged stand. Different letters indicate significant differences among treatments within the same sampling date $(P<0.05)$. 
$\mathrm{kg}^{-1}$ para los arenosos. En la última fecha de muestreo (09/17), luego de 14 meses desde el muestreo inicial, los valores observados de carbono orgánico soluble en suelo fueron significativamente mayores a los valores iniciales $(P<0,05)$ para todos los tratamientos, excepto, para la situación ARC J, donde no se encontraron diferencias significativas. Este incremento fue del $35 \%$ para ARC M, de $66 \%$ y $62 \%$ para ARE J y ARE M, respectivamente. En promedio, los valores de carbono orgánico soluble fueron de $47 \mathrm{mg} \mathrm{kg}^{-1}$ para los suelos arcillosos, y de $39 \mathrm{mg} \mathrm{kg}^{-1}$ para los suelos arenosos, mostrando un incremento respecto a los valores iniciales de $17,5 \%$ y de $62,5 \%$ para los suelos arcillosos y arenosos, respectivamente, siendo esta diferencia estadísticamente significativa solo para los suelos arenosos $(P=0,003)$.

Se encontró una regresión lineal positiva entre el contenido de carbono orgánico soluble y el espacio poroso saturado con agua, y entre las emisiones de $\mathrm{CO}_{2}$ y el contenido de carbono orgánico soluble del suelo (figura 5). La temperatura del aire y la temperatura del suelo no se relacionaron significativamente con los contenidos edáficos de carbono orgánico soluble $(P>0,05)$. Por su parte, las emisiones de $\mathrm{CO}_{2}$ se relacionaron positivamente con la temperatura del suelo $(P<0,001 ; \mathrm{R}=0,36)$.

$\mathrm{Al}$ realizar el análisis de correlación entre las variables edáficas medidas (emisión de $\mathrm{CO}_{2}$, espacio poroso saturado con agua, temperatura del suelo) y el carbono orgánico soluble para suelos arcillosos y arenosos por separado, se encontró una correlación positiva significativa $(P<0,01)$ y entre el contendido de carbono orgánico soluble y la emisión de $\mathrm{CO}_{2}$ tanto para los suelos arcillosos $(\mathrm{R}=0,36)$, como para los arenosos $(\mathrm{R}=0,37)$. Por su parte, la relación entre el carbono orgánico soluble y el espacio poroso sa- turado con agua solo fue significativa $(P<0,01 ; \mathrm{R}=0,56)$ en los suelos arenosos.

Relación entre el mantillo forestal y el carbono orgánico soluble del suelo. El incremento en el carbono orgánico soluble del suelo (carbono orgánico soluble final - carbono orgánico soluble inicial) mostró una asociación positiva y significativa con los valores de materia seca del mantillo total $(P=0,03)$ y del mantillo fino $(P=0,02)$ (figura 6$)$. En

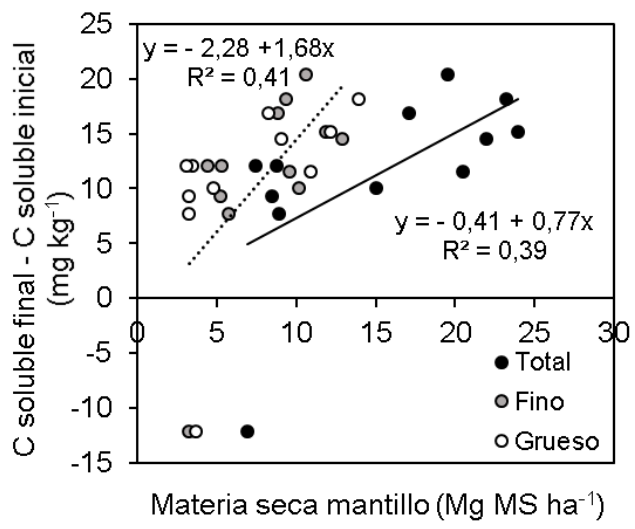

Figura 6. Regresión lineal entre la diferencia entre carbono (C) soluble orgánico inicial y final con la materia seca en el mantillo total, fino y grueso. Las líneas representan la regresión en los casos que fueron significativas (Mantillo total: línea llena; Mantillo fino: línea punteada).

Linear regression between the difference between initial and final soluble organic carbon $(\mathrm{C})$ with the dry matter in the total, fine and coarse litter. The lines represent the regression in the cases that were significant (Total litter: solid line; Fine litter: dotted line).
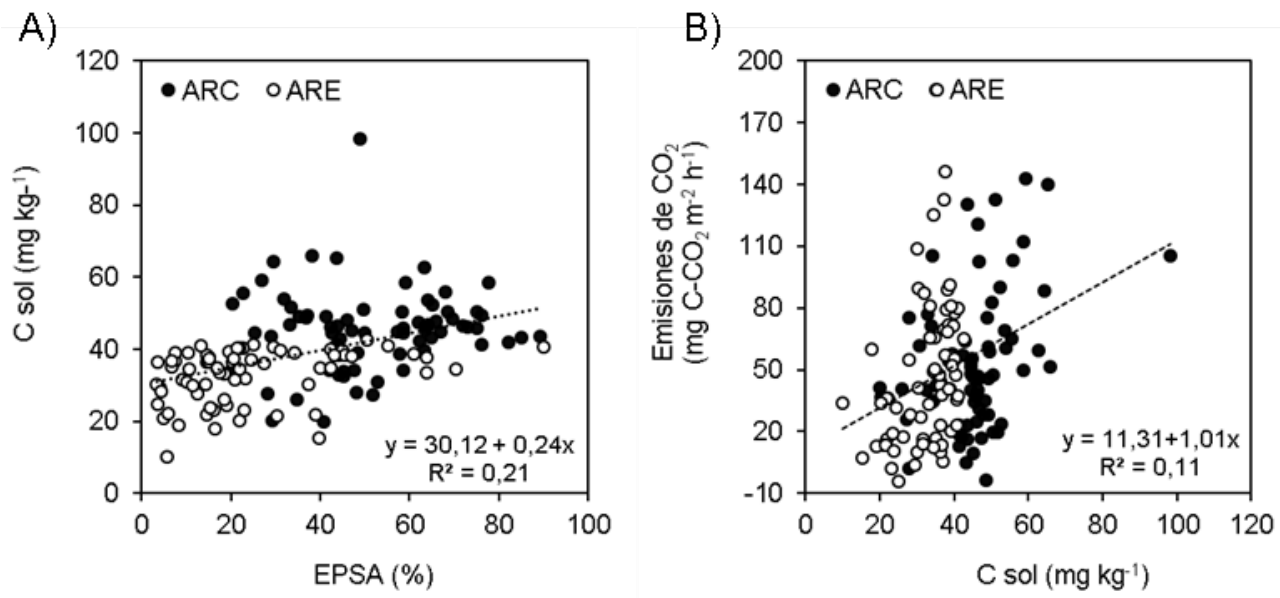

Figura 5. Regresión lineal entre los contenidos de carbono orgánico soluble (C sol) y A) el espacio poroso saturado con agua (EPSA) y B) las emisiones de $\mathrm{CO}_{2}$ desde los suelos. Los símbolos llenos y vacíos corresponden a los suelos de textura arcillosa y arenosa, respectivamente. Las líneas punteadas representan la regresión entre variables. ARC: suelos arcillosos, ARE: suelos arenosos.

Lineal regression among the contents of soluble organic carbon $(\mathrm{C} \mathrm{sol})$ and $\mathrm{A})$ the pore space saturated with water $(\mathrm{EPSA})$ and $\mathrm{B})$ the $\mathrm{CO}_{2}$ emissions from soils. The full and empty symbols correspond to the soils with clayey (ARC) and sandy (ARE) texture, respectively. The dotted lines represent the regression among variables. 
el caso del mantillo grueso la relación no fue estadísticamente significativa $(P=0,07)$.

\section{DISCUSIÓN}

Los valores de materia seca en el mantillo medidos en el presente trabajo se encuentran dentro del rango de los valores observado para plantaciones de E. grandis en Australia, Argentina y Brasil (Goya et al. 1997, Turner y Lambert 2008, Cortez et al. 2014). Estos trabajos reportaron valores entre 2 a $25 \mathrm{Mg} \mathrm{ha}^{-1}$ de materia seca en el mantillo total en plantaciones con edades de entre 1 a 14 años. Estas diferencias en los valores acumulados de mantillo pueden ser atribuidas a las diferentes edades de los rodales, al manejo de las plantaciones y a diferentes condiciones climáticas que actúan sobre su descomposición.

En el presente estudio no se encontraron diferencias significativas en la cantidad de mantillo total entre tratamientos, posiblemente por la gran variabilidad en los datos (figura 2). A pesar de que los valores promedio de materia seca entre ARC J y ARC M presentaron una diferencia absoluta de más de un $50 \%$, las mismas no fueron significativas $(P>0,05)$. La cantidad de muestras de matillo tomadas para caracterizar los sitios pudo no haber sido suficiente para caracterizar la variabilidad espacial propia del sistema. A pesar de la falta de diferencia estadística, pudo observarse que las plantaciones de mediana edad presentaron valores semejantes entre sí y mayores a los encontrados en el rodal joven sobre suelo arcilloso. Estas diferencias son consecuencia de la mayor acumulación de material al aumentar la edad de los rodales. Por su parte, los menores valores de mantillo observados en el tratamiento de rodales jóvenes sobre suelo arcilloso con relación al tratamiento de rodales jóvenes en suelo arenoso se encontrarían asociados a diferencias en la historia previa de los lotes (cuadro 1), ya que este último fue implantado sobre una plantación de pino, quedando los residuos remanentes de la cosecha sobre el suelo, mientras que el resto de las situaciones provenían de campo natural. Esto explica porque los valores de mantillo en el tratamiento de rodales jóvenes sobre suelo arenoso son similares a los de las situaciones maduras, siendo que es la plantación más joven (1 año). Esto indicaría que la historia de uso y manejo puede resultar igual o más importante que condiciones intrínsecas del sitio como la edad de los rodales o tipo de suelo sobre la cantidad mantillo acumulado.

Los contenidos de carbono orgánico total y de carbono orgánico soluble fueron mayores en los suelos arcillosos y presentaron una relación fuerte y positiva con el contenido de arcilla (figura 3). Por el contrario, estos tipos de suelo no presentaron relación con la edad de los rodales. A pesar de que los suelos arcillosos y arenosos presentaron valores similares de materia seca total de mantillo, los mayores contenidos de carbono orgánico en los suelos arcillosos respecto a los arenosos se encontrarían asociados al rol protector de las arcillas sobre el mismo. Según Abramoff et al. (2018) la protección de carbono orgánico total por las partículas de arcillas ocurriría a través de al menos dos mecanismos diferentes. Primero, a medida que el carbono orgánico total se estabiliza químicamente, es adsorbido sobre la superficie de los minerales arcillosos cargados. Segundo, el carbono orgánico total se encuentra físicamente protegido de la mineralización microbiana a través de la formación de agregados del suelo.

Los valores de carbono orgánico soluble observados fueron similares a los informados por Wang et al. (2019 b) en plantaciones de Eucalyptus spp. de Australia bajo condiciones edafoclimáticas similares a las del presente estudio. La relación positiva hallada entre la cantidad de mantillo total y fino, y el incremento de carbono orgánico soluble, estarían indicando que una de las principales fuentes del carbono orgánico soluble en estos suelos sería el carbono liberado mediante la descomposición del mantillo fino (figura 6).

A lo largo del tiempo evaluado, los suelos arcillosos presentaron en todos los casos contenidos de carbono orgánico soluble mayores que los arenosos (figura 4). Según estudios previos realizados en la región mesopotámica por Goya et al. (2008) la tasa anual de descomposición de la materia seca del mantillo fue más rápida en el suelo arcilloso (44 \%) que en los suelos arenosos (30\%), lo que podría asociarse a las mejores condiciones para el desarrollo y actividad de descomponedores microbianos en suelos de textura arcillosa. Esto, sumado a las mínimas pérdidas por lixiviación, conduciría a mayores valores de carbono orgánico soluble en los suelos arcillosos. Sin embargo, cabe destacar que en el presente estudio, no se encontró una correlación significativa entre el contenido de arcilla y la actividad microbiana, estimada a través de la cuantificación de las emisiones de $\mathrm{CO}_{2}$. A su vez, los incrementos en los valores de carbono orgánico soluble luego de 14 meses respecto a los valores iniciales fueron mayores en los suelos arenosos, donde la mayor aireación sumada a la menor protección de la materia orgánica, podrían promover el ataque microbiano del propio humus del suelo, aumentando la liberación de compuestos hidrosolubles.

En el presente estudio y acorde a lo reportado por otros autores (Kalbitz et al. 2000, Cepáková et al. 2016, Wang et al. 2019b), la dinámica del carbono orgánico soluble en el tiempo estuvo principalmente controlada por las condiciones climáticas, aumentando los contenidos de carbono orgánico soluble en los meses de verano (figura 4) cuando las precipitaciones y temperaturas presentaron los mayores valores (figura 1). Acorde al análisis de correlación con las variables edáficas y ambientales cuantificadas, solo presentaron una asociación positiva y significativa con el carbono orgánico soluble, el espacio poroso saturado con agua y la emisión de $\mathrm{CO}_{2}$ (figura 5). Este último permite suponer que mayores contenidos carbono orgánico soluble podrían conducir a mayores emisiones de gases efecto invernadero. Los microorganismos son los agentes primarios responsables de la degradación del mantillo, y consecuentemente, los factores abióticos que afectan la 
actividad microbiana, como la temperatura, la textura del suelo, la densidad aparente, y contenido de humedad también influyen en la descomposición del carbono del mantillo (Silveira et al. 2011). De esta manera, el aumento de la temperatura y las precipitaciones durante los meses estivales incrementan la actividad biológica y por ende aumenta la descomposición de los residuos, lo que conlleva a la ruptura de compuestos orgánicos poco solubles de mayor peso molecular, liberándose compuestos solubles de mayor labilidad que progresivamente se ven incrementados en los primeros centímetros del suelo (Wang et al. 2019b). No obstante que en el presente estudio la actividad de los microorganismos estuvo positivamente asociada con el incremento de la temperatura edáfica, no se halló relación entre el carbono orgánico soluble y la temperatura del aire o suelo. De esta manera la relación positiva hallada entre el carbono orgánico soluble y el espacio poroso saturado con agua indicaría que en los meses más cálidos, cuando la temperatura no es limitante para la actividad biológica, sería el agua contenida en los poros del suelo, el principal factor responsable de los incrementos de carbono orgánico soluble en el suelo. Debido a que los suelos arenosos poseen menor capacidad de retención hídrica que los arcillosos, la actividad microbiana funcionaría a pulsos dependiendo mayormente de las precipitaciones caídas, no ocurriendo lo mismo en los suelos arcillosos, donde el agua es almacenada y conservada en los microporos por un período mayor de tiempo. Esto explicaría el motivo por el cual la relación entre el carbono orgánico soluble y el espacio poroso saturado con agua solo fue significativa para los suelos de textura arenosa.

\section{CONCLUSIONES}

Los contenidos promedio de carbono orgánico soluble en suelos con plantaciones de E. grandis de la región mesopotámica son mayores en los suelos de textura arcillosa $\left(45,6 \mathrm{mg} \mathrm{kg}^{-1}\right)$ que en aquellos de textura arenosos $(32,8 \mathrm{mg}$ $\left.\mathrm{kg}^{-1}\right)$. Estas diferencias están positivamente relacionadas con el contenido de arcillas, pero no se asocian con una mayor actividad biológica en los suelos arcillosos. Cabe destacar que los incrementos en los valores de carbono orgánico soluble medidos 14 meses después del inicio del estudio fueron mayores en los suelos arenosos que en los arcillosos.

La historia de uso y manejo resulta igual o más importante que la edad de los rodales o tipo de suelo sobre la cantidad mantillo acumulado. Por otro lado, se verifica una relación lineal y positiva entre la cantidad de mantillo fino inicial y la acumulación de carbono orgánico soluble en el suelo, evidenciando que una de las principales fuentes del carbono orgánico soluble sería la descomposición del mantillo fino.

Entre los factores evaluados que inciden sobre la actividad biológica, tanto el contenido de arcillas como el espacio poroso saturado con agua se relacionan positivamente con el contenido de carbono orgánico soluble, pre- sentado los suelos arenosos mayor dependencia del contenido de humedad edáfica para la acumulación de carbono orgánico soluble respecto a los suelos arcillosos.

Los resultados hallados aportan magnitudes de las existencias de carbono orgánico total, carbono orgánico soluble y flujos de carbono a nivel local, contribuyendo con información necesaria para el modelado del carbono en sistemas forestales.

\section{AGRADECIMIENTOS}

Esta investigación fue financiada por el proyecto de Investigación Aplicada PIA 14085 (BID 405 programa 2853/OC-AR). Los autores agradecen al establecimiento rural "El potrero de San Lorenzo".

\section{REFERENCIAS}

Abramoff R, X Xu, M Hartman, S O'Brian, W Feng, E Davidson, A Finzi, D Moorhead, J Schimel, M Torn, MA Mayes. 2018. The Millennial model: in search of measurable pools and transformations for modeling soil carbon in the new century. Biogeochemistry 137 (1): 51-71. DOI: https://doi. org/10.1007/s10533-017-0409-7

Ashworth J, D Keyes, R Kirk, R Lessard. 2001. Standard Procedure in the Hydrometer Method for Particle Size Analysis. Communications in Soil Science and Plant Analysis 32: 633- 642. DOI: https://doi.org/10.1081/CSS-100103897

Cepáková S, Z Tošner, J Frouz. 2016. The effect of tree species on seasonal fluctuations in water-C solubleand hot waterextractable organic matter at post-mining sites. Geoderma 275:19-27. DOI: https://doi.org/10.1016/j.geoderma.2016.04.006

Chen CR, ZH Xu. 2005. Soil carbon and nitrogen pools and microbial properties in a 6-year-old slash pine plantation of subtropical Australia: impacts of harvest residue management. Forest Ecology and Management 206 (1-3): 237247. DOI: https://doi.org/10.1016/j.foreco.2004.11.005

Cortez CT, L Nunes, LB Rodrigues, N Eisenhauer, ASFAraujo. 2014. Soil microbial properties in Eucalyptus grandis plantations of different ages. Journal of Soil Science and Plant Nutrition 14 (3): 734-742. DOI: https://doi.org/10.4067/ S0718-95162014005000059

De Lucena MS, AR Alves, I Alves Bakke. 2019. Aporte de mantillo y nutrientes en ecosistema semiárido de Brasil (Caatinga) bajo sistemas silvícolas. Madera y Bosques 25 (1): 1-16. DOI: https://doi.org/10.21829/myb.2019.2511597

Forte Lay JA, OE Scarpati, AD Capriolo. 2008. Precipitation variability and soil water content in Pampean Flatlands (Argentina). Geofisica Internanional 47 (4): 341-354. DOI: https://doi.org/10.22201/igeof.00167169p.2008.47.4.2146

Goya JF, JL Frangi, F Dalla Tea, MA Marcó, F Larocca. 1997. Biomasa, productividad y contenido de nutrientes en plantaciones de Eucalyptus grandis en el NE de la Provincia de Entre Ríos. In XII Jornadas Forestales de Entre Ríos. Concordia, Argentina. p. III-1-19.

Goya JF, JL Frangi, C Pérez, F Dalla Tea. 2008. Decomposition and nutrient release from leaf litter in Eucalyptus grandis plantations on three different soils in Entre Ríos, Argenti- 
na. Bosque 29(3): 217-226. DOI: https://doi.org/10.4067/ $\underline{\mathrm{s} 0717-92002008000300005}$

R.J Haynes RJ. 2000. Labile organic matter as an indicator of organic matter quality in arable and pastoral soils in New Zealand. Soil Biology and Biochemistry 32 (2) 211-219. DOI: https://doi.org/10.1016/S0038-0717(99)00148-0

Hättenschwiler S, AV Tiunov, S Scheu. 2005. Biodiversity and litter decomposition in terrestrial ecosystems. Annual Review of Ecology, Evolution, and Systematics 36:191-218. DOI: https://doi.org/10.1146/annurev.ecolsys.36.112904.151932

Hofman J, L Dušek. 2003. Biochemical analysis of soil organic matter and microbial biomass composition - a pilot study. European Journal of Soil Biology 39 (4): 217-224. DOI: https://doi.org/10.1016/j.ejsobi.2003.08.002

IPCC. 2006. Guidelines for National Greenhouse Gas inventories. Eds. Eggleston HS, L Buendia, K Miwa, T Ngara, K Tanabe. Institute for Global Environmental Strategies. Hayama, Japan.

Kalbitz K, S Solinger, JH Park, B Michalzik, E Matzner. 2000. Controls on the Dynamics of Dissolved Organic Matter in Soils: A Review. Soil Science 165 (4): 277-304. DOI: https://doi.org/10.1097/00010694-200004000-00001

Leifeld J, I Kögel-Knabner. 2005. Soil organic matter fractions as early indicators for carbon stock changes under different land use. Geoderma 124(1-2): 143-155. DOI: https://doi. org/10.1016/j.geoderma.2004.04.009

MAGyP (Ministerio de Agricultura, Ganadería y Pesca, AR). 2020. Inventario nacional de plantaciones forestales por superficie. Consultado 23 de sep. 2020. Disponible en https:// datos.agroindustria.gob.ar/dataset/inventario-nacionalplantaciones-forestales-por-superficie

McLauchlan KK. 2006. Effects of soil texture on soil carbon and nitrogen dynamics after cessation of agriculture. Geoderma 136(1-2): 289-299. DOI: https://doi.org/10.1016/j.geoderma.2006.03.053.

Millar N, EM Baggs. 2004. Chemical composition, or quality, of agroforestry residues influences $\mathrm{N}_{2} \mathrm{O}$ emissions after their addition to soil. Soil Biology and Biochemistry 36: 935943. DOI: $\underline{\text { https://doi.org/10.1016/j.soilbio.2004.02.008 }}$

Montero G, R Ruiz-Peinado, M Muñoz. 2005. Producción de biomasa y fijación de $\mathrm{CO}_{2}$ por los bosques españoles. Monografías INIA: Serie Forestal nº 13. Madrid, España. Instituto Nacional de Investigación y Tecnología Agraria y Alimentaria, Ministerio de Educación y Ciencia. 270 p.
Nelson DW, LE Sommers. 1996. Total carbon, organic carbon, and organic matter. In Sparks DL, AL Page, PA Helmke, RH Loeppert, PN Soltanpour, MA Tabatabai, CT Johnston, ME Sumner eds. Methods of Soil Analysis. Part 3, Soil Science Society of America Book Series, Madison. p 9611010. DOI: https://doi.org/10.2136/sssabookser5.3.c34

Sayer EJ. 2006. Using experimental manipulation to assess the roles of leaf litter in the functioning of forest ecosystems. Biological Reviews 81(1):1-31. DOI: https://doi. org/10.1017/S1464793105006846

Silveira ML, KR Reddy, NB Comerford. 2011. Litter Decomposition and $\mathrm{C}$ soluble Carbon, Nitrogen, and Phosphorus Release in a Forest Ecosystem. Open Journal of Soil Science 1: 86-96. DOI: https://doi.org/10.4236/ojss.2011.13012

Soil Survey Staff. 2014. Keys to Soil Taxonomy, $12^{\text {th }}$ ed. USDANatrual Resources Conservation Service, Washington, DC.

Turner J, MJ Lambert. 2008. Nutrient cycling in age sequences of two Eucalyptus plantation species. Forest Ecology and Management 255(5-6): 1701-1712. DOI: https://doi. org/10.1016/j.foreco.2007.11.038

Van Diepen LTA, SD Frey, CM Sthultz, EW Morrison, R Minocha, A Pringle. 2015. Changes in litter quality caused by simulated nitrogen deposition reinforce the $\mathrm{N}$-induced suppression of litter decay. Ecosphere 6(10):1-15. DOI: https://doi.org/10.1890/ES15-00262.1

Vance ED, PC Brookes, DS Jenkinson. 1987. An extraction method for measuring soil microbial biomass C. Soil Biology and Biochemistry 19(6): 703-707. DOI: https://doi. org/10.1016/0038-0717(87)90052-6

Wang M, Q Tian, C Liao, R Zhao, D Wang, Y Wu, Q Li, X Wang, F Liu. 2019a. The fate of litter-derived dissolved organic carbon in forest soils: results from an incubation experiment. Biogeochemistry 144: 133-147. DOI: https://doi. org/10.1007/s10533-019-00576-3

Wang Y, J Zheng, Z Xu, KM Abdullah, Q Zhou. 2019b. Effects of changed litter inputs on soil labile carbon and nitrogen pools in a eucalyptus-dominated forest of southeast Queensland, Australia. Journal of Soils and Sediments 19: 1661-1671. DOI: https://doi.org/10.1007/s11368-019-02268-9

Zou XM, HH Ruan, Y Fu, XD Yang, LQ Sha. 2005. Estimating soil labile organic carbon and potential turnover rates using a sequential fumigation-incubation procedure. Soil Biology and Biochemistry 37 (10): 1923-1928. DOI: https://doi. org/10.1016/j.soilbio.2005.02.028 
